\title{
EFEITO DOS FATORES DE CRESCIMENTO NA CICATRIZAÇÃO DO PÉ DIABÉTICO: UMA REVISÃO DE LITERATURA
}

\section{EFFECT OF GROWTH FACTORS ON DIABETIC FOOT HEALING: A LITERATURE REVIEW}

Recebido em: 02/06/2018.

Aceito em: 28/11/2018.

\author{
Tainá de Lelis Lopes de Carvalho² \\ Nayara Alves de Sousa ${ }^{3}$ \\ Marcia Meira Guimarães ${ }^{4}$ \\ Zâmia Aline Barros Ferreira ${ }^{5}$ \\ Livia Mara Gomes Pinheiro 6
}

\section{RESUMO}

O objetivo deste estudo foi revisar a literatura sobre a ação e eficácia dos fatores de crescimento na cicatrização do pé diabético. Trata-se de uma revisão bibliográfica realizada mediante a busca de artigos científicos no Sistema Online de Busca e Análise de Literatura do serviço da National Library of Medicine dos Estados Unidos da América, publicados entre os anos de 2012 a 2018. Os artigos foram selecionados na língua inglesa, e que relatassem sobre o uso da técnica de fatores de crescimento de forma tópica ou injetável em humanos. Para o reconhecimento dos artigos foram utilizados os termos fatores de crescimento, cicatrização e pé diabético. Foi utilizado o operador boleano "and" para realizar o cruzamento das palavras chave no banco de dados. Foram encontrados nove artigos obtidos pela busca que foram organizados e selecionados em um quadro contendo autor/ano, objetivo e resultados. A análise dos

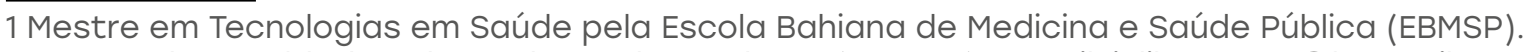
Docente da Faculdade Independente do Nordeste (FAINOR). E-mail: julibarros78@hotmail.com

2 Graduada em Estética e Cosmética pela Faculdade Independente do Nordeste (FAINOR). E mail: tainalelis_gv@hotmail.com

3 Doutora em Desenvolvimento e Meio Ambiente pela Universidade Estadual de Santa Cruz (UESC). Docente do da Universidade Estadual do Sudeste da Bahia (UESB).

E-mail: nayara.sousa1@hotmail.com

4 Mestre em Meio Ambiente pela Universidade Estadual de Santa Cruz (UESC). Docente da Faculdade Independente do Nordeste (FAINOR). E-mail: marciameira@fainor.com.br

5 Mestre em Tecnologias em Saúde pela Escola Bahiana de Medicina e Saúde Pública (EBMSP). Docente da Faculdade Independente do Nordeste (FAINOR). E mail zamialine@hotmail.com

6 Mestre em Ética e Gestão pela Escola Superior de Teologia (Faculdades EST). Docente da Faculdade Independente do Nordeste (FAINOR). E mail liviamara@fainor.com.br 
artigos mostrou que há, em sua maioria, resultados satisfatórios com o uso dos fatores de crescimento na cicatrização de feridas do pé diabético. Foi concluído que os fatores de crescimento são eficazes no tratamento do pé diabético.

Palavras-chave: Fatores de crescimento. Cicatrização. Pé diabético.

\section{ABSTRACT}

The objective of this study was to review the literature on the action and efficacy of growth factors on diabetic foot healing. This is a bibliographical review carried out through the search of scientific articles in the Online System of Search and Analysis of Literature of the service of the National Library of Medicine of the United States of America, published between the years of 2012 to 2018. The articles were selected in the and reported on the use of the growth factor technique in a topical or injectable form in humans. For the recognition of the articles were used the terms growth factors, healing and diabetic foot. The Boolean operator "and" was used to cross-reference the keywords in the database. We found nine articles obtained by the search that were organized and selected in a table containing author / year, objective and results. The analysis of the articles showed that there are, for the most part, satisfactory results with the use of growth factors in the healing of diabetic foot wounds. It was concluded that growth factors are effective in the treatment of diabetic foot.

Keywords: Growth factors. Healing. Diabetic foot.

\section{INTRODUÇÃO}

O diabetes mellitus (DM) é definido como uma deficiência no momento da produção e/ou excreção da insulina (hormônio produzido pelo pâncreas) o que estabelece um alto nível de glicose no sangue (YANG et al., 2016). Dados estatísticos mostram que aproximadamente 347 milhões de pessoas no mundo têm DM e em 2030 este número tende a aumentar para 353 milhões (MENEZES et al., 2016; GOMEZ et al., 2014).

Estudos atuais indicam que mais de 125 milhões de pessoas irão apresentar complicação do diabetes, e 25\% dos indivíduos apresentarão uma ulceração nos pés durante a vida (OJALVO et al., 2017). Isso ocorre porque o pé diabético (PD) é uma complicação do DM e a maior causa de amputações de membros inferiores (OLIVEIRA et al., 2013; BRASIL, 2016; SRIDHARAN, SIVARAMAKRISHNAN 2017; ERTUGRUL et al., 2017).

O PD se caracteriza pela situação de infecção, ulceração ou também destruição dos tecidos profundos dos pés, associada a anormalidades neurológicas e vários graus 
de doença vascular periférica, nos membros inferiores de pacientes com DM (PARISI, 2018). A etiologia é multifatorial e o processo de cicatrização é dificultado por fatores como inflamação crônica, defeitos na função dos fibroblastos e da angiogênese, falha na migração celular, associado a isso, tem-se a falta de oxigenação dos membros inferiores (MMII), que gera uma isquemia, um fator crítico na resistência da cicatrização (ELISAVET et al., 2012).

Mesmo com os tratamentos existentes para o PD, estão sendo utilizados novos modelos de intervenções na cicatrização de feridas, como a tecnologia dos fatores de crescimento (FTC's) (MARTÍ et al., 2015). Esses são agentes biológicos produzidos no nosso organismo, que auxiliam na formação de novas células e cada FTC's é responsável por uma atividade como: angiogênese, proliferação celular; fibroblástica e endotelial, síntese de queratinócitos, regeneração nervosa periférica, auxílio no controle do estresse oxidativo, síntese de matriz extracelular e colágeno, entre outras funções (PICARD et al., 2015).

O estudo de MARTÍ et. al., 2015 mostra que pacientes com úlceras diabéticas possuem déficit de fatores de crescimento se comparados a úlceras não diabéticas, mas mesmo com a falta desses elementos no organismo, os FTC's podem ser estimulados ou alterados na indústria para que aumente a síntese de tecido de granulação e preceda a cicatrização no interior da ferida.

Em razão dos elevados índices de ocorrência das lesões no PD, e da associação com outras patologias, estas feridas tornaram-se um problema de saúde pública, além de gerar um alto custo de tratamento, e diminuir a qualidade de vida do indivíduo (OJALVO et al., 2017).

O PD representa uma das mais frequentes e incapacitantes complicações crônicas advindas do mau controle do diabetes que possui alta taxa de amputação. Portanto, frente a este contexto, o objetivo desse estudo que é revisar a literatura sobre a ação e eficácia dos fatores de crescimento na cicatrização do pé diabético.

\section{METODOLOGIA}

Foi realizado um estudo de revisão bibliográfica entre os meses de março a maio de 2018 uma busca de artigos científicos publicados entre os anos de 2012 a 2018 , nos idiomas português e inglês.

Os critérios de inclusão foram os artigos que relatassem sobre o uso da técnica de fatores de crescimento de forma tópica ou injetável em humanos. Os critérios de exclusão foram artigos que utilizaram outras formas de tratamento associados aos fatores de crescimento, como câmara hiperbárica, alta frequência, ultrassom (fonoforese), aplicação ou uso em animais e artigos não disponíveis na íntegra.

Os artigos foram encontrados, na plataforma eletrônica de pesquisa científica: no Sistema Online de Busca e Análise de Literatura do serviço da National Library of Medicine dos Estados Unidos da América (PUBMED). Todos os artigos escolhidos 
abordavam sobre o uso da técnica de fatores de crescimento de forma tópica ou injetável em humanos.

As palavras-chave utilizadas individualmente, ou em associação, utilizadas foram: Fatores de crescimento, Cicatrização, Pé diabético (Growth Factors, Healing, Diabetic Foot). Foi utilizado o operador boleano "and" para realizar o cruzamento das palavras chave no banco de dados.

Foram encontrados quatrocentos e quarenta artigos científicos que tratavam sobre o assunto, porém, de acordo com os critérios de inclusão foram selecionados sessenta e nove artigos. Após a leitura dos resumos e análise, permaneceram nove artigos (Nível de Evidência Científica I e II) que serviram de base para esta pesquisa.

\section{RESULTADOS E DISCUSSÃO}

Foram selecionados 9 estudos, sendo: 7 revisões sistemáticas, 1 estudo experimental e 1 estudo multicêntrico. A análise dos artigos mostrou que há em sua maioria, resultados satisfatórios com o uso dos fatores de crescimento na cicatrização de feridas do pé diabético.

Após a escolha e tradução dos artigos foi realizado um resumo com o intuito de melhor entendimento sobre o assunto e junção com a pesquisa. Em seguida, foi realizada uma divisão entre os artigos separando autor/ ano, objetivo, protocolo e resultados, sendo colocados organizadamente, de forma crescente por ano de publicação (QUADRO 1).

Quadro 1: Características dos estudos selecionados sobre a ação dos fatores de crescimento na cicatrização do pé diabético

\begin{tabular}{|c|c|c|c|}
\hline Autor/Ano & Objetivo & Protocolo & Resultados \\
\hline ELISAVET et al., 2012. & $\begin{array}{c}\text { Avaliar o efeito do } \\
\text { Fator de crescimento } \\
\text { epidérmico (EGF) na } \\
\text { cicatrização de úlceras do } \\
\text { pé diabético. }\end{array}$ & $\begin{array}{l}\text { Foi utilizado o creme } \\
\text { de EGF a 0,04\% duas } \\
\text { vezes ao dia A injeção } \\
\text { intralesional foi utilizada } \\
\text { posologia de } 75 \mu \mathrm{g} \text { de } \\
\text { EGF, três vezes por } \\
\text { semana. }\end{array}$ & $\begin{array}{l}\text { O EGF auxiliou de forma } \\
\text { eficaz na cicatrização do } \\
\text { PD, atuando em células } \\
\text { epiteliais e fibroblastos } \\
\text { restaurando o epitélio } \\
\text { danificado. }\end{array}$ \\
\hline GOMEZ et al., 2014. & $\begin{array}{l}\text { Determinar a eficácia } \\
\text { clínica e segurança do } \\
\text { rhEGF no aumento da } \\
\text { cicatrização da ferida } \\
\text { diabética e tecido de } \\
\text { granulação }\end{array}$ & $\begin{array}{l}\text { O rhEGF Foi utilizado } \\
\text { três vezes por } \\
\text { semana, com aplicação } \\
\text { intralesional, posologia } \\
\text { de } 75 \mu \mathrm{g} \text {, e o grupo } \\
\text { placebo utilizou PD por } \\
8 \text { semanas. A posologia } \\
\text { e intervalo de aplicação } \\
\text { foram os mesmos. }\end{array}$ & $\begin{array}{l}\text { Resultados mostraram } \\
\text { que em pacientes com } \\
\text { PD que receberam } \\
\text { tratamento padrão, } \\
\text { aplicação rhEGF } \\
\text { intralesional resultou } \\
\text { em cicatrização } \\
\text { completa promovendo } \\
\text { a epitelização do leito } \\
\text { da ferida e reduzindo } \\
\text { significativamente a área } \\
\text { da ferida tratada. }\end{array}$ \\
\hline
\end{tabular}




\begin{tabular}{|c|c|c|c|}
\hline ZHAO et al., 2014. & $\begin{array}{l}\text { O objetivo deste estudo } \\
\text { foi realizar uma revisão } \\
\text { sistemática atualizada e } \\
\text { meta-análise para avaliar } \\
\text { a eficácia clínica da } \\
\text { rhPDGF no tratamento } \\
\text { de úlceras diabéticas de } \\
\text { extremidade inferior. }\end{array}$ & $\begin{array}{l}\text { Um total de } 6 \text { ensaios } \\
\text { clínicos randomizados, } \\
\text { incluindo } 992 \text { pacientes, } \\
\text { foi selecionado de } 173 \\
\text { estudos identificados. } \\
\text { Uma média de } \\
\text { tratamento entre } 10 \text { a } \\
20 \text { semanas. A posologia } \\
\text { não foi bem apresentada, } \\
\text { sendo de forma tópica. }\end{array}$ & $\begin{array}{c}\text { Os resultados indicam } \\
\text { que a rhPDGF é eficaz } \\
\text { no tratamento das ulceras } \\
\text { diabeticas dos membros } \\
\text { inferiores. Auxiliando na } \\
\text { cicatrização. }\end{array}$ \\
\hline MARTÍ et al., 2015. & $\begin{array}{l}\text { Avaliar os benefícios e } \\
\text { malefícios dos fatores } \\
\text { de crescimento em } \\
\text { pacientes com ulcera do } \\
\text { pé diabético com diabetes } \\
\text { mellitus tipo } 1 \text { ou tipo } 2 .\end{array}$ & $\begin{array}{c}\text { A frequência da } \\
\text { administração variou } \\
\text { entre uma vez por } \\
\text { dia até três vezes por } \\
\text { semana com duração } \\
\text { de seis a doze semanas } \\
\text { para a cicatrização com } \\
\text { posologias entre } 75 \mu \mathrm{g} \text { e } \\
25 \mu \mathrm{g} \text {. }\end{array}$ & $\begin{array}{c}\text { O tratamento do pé } \\
\text { diabético utilizando } \\
\text { fatores de crescimento, } \\
\text { quando comparado com } \\
\text { placebo tornaram a } \\
\text { cicatrização completa das } \\
\text { úlceras com resultados } \\
\text { mais favoráveis. }\end{array}$ \\
\hline PICARD et al., 2015. & $\begin{array}{c}\text { Resumir o conhecimento } \\
\text { baseado em evidências } \\
\text { sobre o tratamento } \\
\text { de feridas crônicas } \\
\text { diabéticas por Plasma } \\
\text { rico em plaquetas. }\end{array}$ & $\begin{array}{l}\text { Revisão sistemática } \\
\text { realizada com estudos } \\
\text { que avaliaram o efeito } \\
\text { clínico do plasma rico em } \\
\text { plaquetas na cicatrização } \\
\text { de doenças crônicas } \\
\text { feridas foram incluídas. }\end{array}$ & $\begin{array}{c}87,5 \% \text { dos estudos } \\
\text { controlados encontraram } \\
\text { um benefício significativo } \\
\text { para a adjunção do } \\
\text { plasma rico em plaquetas } \\
\text { tratar feridas diabéticas } \\
\text { crônicas. Como o PRP } \\
\text { pode ser benéfico, } \\
\text { sugerimos o uso de PRP } \\
\text { em úlceras. }\end{array}$ \\
\hline YANG et al., 2016. & $\begin{array}{l}\text { O objetivo do presente } \\
\text { estudo foi realizar uma } \\
\text { revisão sistemática e } \\
\text { metanálise avaliar a } \\
\text { eficácia clínica do rhEGF } \\
\text { no tratamento de úlceras } \\
\text { do pé diabético. }\end{array}$ & $\begin{array}{l}\text { Revisão sistemática } \\
\text { sobre a eficácia clínica do } \\
\text { rhEGF no tratamento de } \\
\text { úlceras do pé diabético. }\end{array}$ & $\begin{array}{c}\text { Estes resultados indicam } \\
\text { que o rhEGF é eficaz } \\
\text { no tratamento de } \\
\text { diabetes úlceras do pé, } \\
\text { aumentando a taxa de } \\
\text { cicatrização de feridas. } \\
\text { Estas descobertas } \\
\text { suportam o uso de } \\
\text { rhEGF no tratamento do } \\
\text { pé diabético. }\end{array}$ \\
\hline ERTUGRUL et al., 2017. & $\begin{array}{c}\text { Analisar os resultados de } \\
\text { pacientes com úlceras do } \\
\text { pé diabético tratadas com } \\
\text { EGF intralesional. }\end{array}$ & $\begin{array}{l}\text { 174 pacientes de } 25 \\
\text { centros médicos turcos } \\
\text { foram avaliados. Os } \\
\text { pacientes receberam } \\
\text { injeções intralesional } \\
\text { de EGF três vezes por } \\
\text { semana. A mediana da } \\
\text { cicatrização foi de } 4 \\
\text { semanas. }\end{array}$ & $\begin{array}{l}\text { Os pacientes com úlcera } \\
\text { do pé diabético que } \\
\text { receberam tratamento } \\
\text { padrão a aplicação de } \\
\text { EGF intralesional, após } \\
\text { o controle de infecção, } \\
\text { apresentaram taxas de } \\
\text { cura e baixas taxas de } \\
\text { amputação. }\end{array}$ \\
\hline
\end{tabular}




\begin{tabular}{|c|c|c|c|}
\hline OJALVO et al., 2017. & $\begin{array}{l}\text { Caracterizar a resposta } \\
\text { de pacientes com PD ao } \\
\text { tratamento do fator de } \\
\text { crescimento epidérmico } \\
\text { em termos de marcadores } \\
\text { de status redox. }\end{array}$ & $\begin{array}{c}\text { Treze pacientes com } \\
\text { diabetes mellitus tipo } \\
1 \text { e } 2 \text { foram submetidos } \\
\text { a } 75 \mu \text { g de EGF a cada } \\
\text { infiltração intra-lesional } \\
\text { sessão, três vezes } \\
\text { por semana, em dias } \\
\text { alternados. }\end{array}$ & $\begin{array}{c}\text { Os resultados } \\
\text { indicam que o fator de } \\
\text { crescimento epidérmico } \\
\text { (EGF) a terapia intra- } \\
\text { úlcera contribui para } \\
\text { restaurar o equilíbrio } \\
\text { redox sistêmico em } \\
\text { pacientes com PD. }\end{array}$ \\
\hline $\begin{array}{c}\text { SRIDHARAN e } \\
\text { IVARAMAKRISHNAN, } \\
2017 .\end{array}$ & $\begin{array}{l}\text { Avaliar se os fatores } \\
\text { tópicos de crescimento } \\
\text { aceleram a cicatrização de } \\
\text { feridas em pacientes com } \\
\text { úlceras do pé diabético. }\end{array}$ & $\begin{array}{l}\text { Revisão sistemática sobre } \\
\text { fatores de crescimento } \\
\text { com via tópica. Foram } \\
\text { realizados com } 26 \\
\text { estudos, com } 2088 \\
\text { participantes e } 1018 \\
\text { artigos incluídos. }\end{array}$ & $\begin{array}{c}\text { Os fatores rhEGF, } \\
\text { rhPDGF e PRP } \\
\text { autólogo melhoraram } \\
\text { significativamente a } \\
\text { taxa de cicatrização } \\
\text { quando utilizados como } \\
\text { adjuvantes para o padrão } \\
\text { de cuidados, sendo que } \\
\text { o rhEGF pode ter um } \\
\text { melhor desempenho } \\
\text { que outros fatores de } \\
\text { crescimento. Os achados } \\
\text { podem não ser aplicáveis } \\
\text { para PD com infecção ou } \\
\text { osteomielite. }\end{array}$ \\
\hline
\end{tabular}

Fonte: Dados da Pesquisa, 2018.

Foram citados na pesquisa os seguintes fatores de crescimento: fator de crescimento epidérmico (EGF); fator de crescimento recombinante humano (rhEGF); fator de crescimento endotelial vascular (VEGF); fator de crescimento placentário (PIGF); fator de crescimento derivado de plaquetas recombinante humano (rhPDGF$\mathrm{BB})$; fator de crescimento de plasma rico em plaquetas (PRP); fator de crescimento transformador $\beta 2$ (TGF- $\beta 2$ ); fator de crescimento de fibroblastos básico humano recombinante (FGFb); fator de crescimento endotelial vascular humano recombinante (VEGF); fator de crescimento de fibroblastos acídico humano recombinante (AFGF) e peptídeo de ácido arginina-glicina-aspártico matriz (RGD).

Elizavet et al., 2012 trouxeram em uma revisão sistemática a conclusão de que o uso tópico de EGF a 0,04\% utilizado duas vezes ao dia, leva seis semanas para a cicatrização da lesão no PD. Essa é a forma mais eficaz quando comparada a outras posologias e veículos a serem utilizados de forma tópica. Tratando-se da injeção intralesional, a forma mais eficaz foi com posologia de $75 \mu \mathrm{g}$ de EGF, três vezes por semana.

A injeção intralesional foi considerada a forma mais eficaz para o tratamento do PD devido a sua biodisponibilidade ser melhor que a forma tópica, em função do ativo poder chegar ao tecido de uma forma mais efetiva. 50\% da ferida apresentou tecido de granulação em duas semanas. Foi evidenciado, também, efeito favorável para diminuição na taxa de amputação. O efeito adverso da forma tópica é irritação da pele enquanto da forma intralesional é a dor durante a aplicação. Os autores julgam ser eficaz a utilização 
do EGF na cicatrização do PD, porém, não descartam o uso das terapias tradicionais no tratamento do PD, por ter sido observado que pode aparecer novas lesões.

Gomez et al., 2014, em um ensaio clínico randomizado, mostram o efeito do rhEGF. Esse fator é um polipeptídeo responsável pela angiogênese, que estimula a formação da matriz extracelular, a proliferação celular, além de promover citoproteção de oxidação. O rhEGF foi utilizado três vezes por semana, com aplicação intralesional com posologia de $75 \mu \mathrm{g}$ comparado com o placebo, durante 8 semanas. A posologia e intervalo de aplicação foram os mesmos.

Os resultados não foram tão satisfatórios quanto aos achados na literatura. Independente dessa variação o resultado na cicatrização foi eficaz, mesmo em pacientes nos quais a ferida não foi fechada por completo. Os autores mostraram que a lesão foi diminuída, e houve formação de tecido de granulação, e alertam ainda que, neste estudo as feridas isquêmicas não foram avaliadas e tratadas.

Zhao et al., 2014 em outra revisão sistemática, com 6 ensaios clínicos randomizados, realizados em 992 pacientes, mostraram resultados da comparação do tratamento utilizando o rhPDGF com tratamento placebo. Nesta pesquisa não foi relatada de forma explícita a posologia no tratamento, nem a quantidade de sessões, mas de forma geral foi visto que a aplicação tópica foi de $100 \mu \mathrm{g} / \mathrm{g}$ e $30 \mu \mathrm{g} / \mathrm{g}$ com a média de tempo de cicatrização de 10 a 20 semanas.

A utilização do fator rhPDGF associado aos cuidados padrões da ferida (debridamento, cuidados com feridas locais- higienização para troca de curativo, controle da infecção e descarga de pressão- com sapatos adequados) obtiveram benefícios para a cicatrização do PD. Assim como Yang et al., 2016, estes autores afirmam que a utilização de rhPDGF, apresentam efeitos adversos como: infecção e celulite, além de edema periférico, porém, é uma técnica segura e eficaz.

Martí et al., 2015 realizaram uma revisão sistemática de 28 ensaios clínicos randomizados, com uma população total de 2365 participantes com DM tipo 1 e 2 . Nesta revisão, os autores encontraram 11 diferentes FTC's e a via tópica foi à forma mais frequente de administração, somente dois estudos utilizaram a injeção via intralesional. A frequência da administração variou entre uma vez por dia, até três vezes por semana com duração de seis a doze semanas para a cicatrização, com posologias entre $25 \mu \mathrm{g}$ e $75 \mu \mathrm{g}$.

A comparação de qualquer FTC com placebo mostra que os FTC aumentam a probabilidade para obter uma cicatrização completa da ferida. Já a comparação dos FTC's com o colágeno oxidado não apresenta dados concretos e satisfatórios. Os resultados deste estudo mostraram uma maior capacidade de cicatrização para os pacientes expostos aos FTC's, porém não houve evidências quanto à diminuição dos níveis de amputação.

A revisão sistemática realizada por Picard et al., 2015, mostra os resultados baseados no PRP's. 12 estudos utilizaram como protocolo o PRP em forma de gel com aplicação tópica. 41,7\% dos estudos utilizaram um protocolo com duas aplicações por 
semana e 33,3\% utilizaram aplicação uma vez por semana. Somente um artigo utilizou um protocolo com uma aplicação por dia, durante 20 semanas. Foram encontrados efeitos adversos: uma dermatite de contato e três cicatrizes.

Como resultado geral, considerou-se que o PRP foi eficaz na aceleração da cicatrização completa. De acordo com esta pesquisa o fator PRP mostrou-se benéfico e mais eficaz em relação aos tratamentos padrões, comparado ao tempo de cicatrização, além de ser um tratamento de baixo custo e de fácil preparação.

Yang et al., 2016 em outra revisão sistemática com meta- análise, utilizou 4 ensaios clínicos randomizados sobre o rhEGF. Neste estudo foram incluídos 294 pacientes com úlceras no $\mathrm{PD}$, destes 185 foram tratados com rhEGF e 109 participantes foram tratados como placebo, todos os participantes possuíam neuropatia diabética. Como resultado foi confirmado que o rhEGF é eficaz na cicatrização das feridas do PD e aumenta a taxa de cicatrização, independente da forma de administração.

Estes resultados estão em conformidade com os pesquisadores Gomez et.al., 2014, Martí et al., 2015 e Sridharan e Sivaramakrishnan, 2017. A divergência é apenas quanto aos efeitos adversos que, para Yang et. Al., 2016 ocasionam celulite, osteomelite e infecção da ferida.

Ertugrul et al., 2017 realizou um estudo retrospectivo, multicêntrico, realizado em 25 centros médicos turcos, com o total de 174 pacientes com diabetes mellitus tipo I e II, e assim como Elizavet et al., 2012, defende o uso do EGF de forma intralesional com posologia de $75 \mu \mathrm{g}$ de EGF, três vezes por semana em dias alternados. O EGF foi mantido refrigerado em temperaturas entre $48{ }^{\circ} \mathrm{C}$ a $88{ }^{\circ} \mathrm{C}$, sendo diluído com 5 ml de água destilada para injeção. A frequência média de tratamento foi de quatro semanas com 12 doses de EGF, e obtiveram $75 \%$ de tecido de granulação ou fechamento completo da lesão. No final do tratamento, 81 pacientes tiveram a cicatrização com a utilização apenas do EGF.

Estes autores ainda concordam que o EGF é eficaz na diminuição das taxas de amputação, e também no aparecimento de lesões pós-tratamento, e por esse motivo é indicado o uso auxiliar das terapias cotidianas: drogas antimicrobianas, debridamento, oxigênioterapia hiperbárica. Além de descreverem que essas taxas reduzidas ocorrem após o controle da infecção, para dar início à aplicação da injeção intralesional. Nesta pesquisa, foi evidenciada a inclusão de pacientes com PD e com insuficiência renal, e como resultado, mostrou ser um tratamento confiável para esse grupo de pacientes.

Diferente de Elizavet et al., 2012, Ertugrul, et al., 2017 encontrou outros efeitos adversos durante a utilização do EGF: calafrios/ tremores $(37,9 \%)$, náusea $(22,9 \%)$, infecção no local da aplicação (4,6\%), síncope $(1,7 \%)$ e dificuldade respiratória $(0,6 \%)$.

Ojalvo et al., 2017 realizaram um estudo experimental com treze pacientes, diagnosticados com diabetes mellitus tipo 1 e 2 , sendo o tipo 2 prevalente. Todos os participantes apresentavam úlceras neuropáticas dos MMII, classificadas como moderadas a graves. O tratamento foi realizado com injeção intralesional de EGF, aplicado três vezes por semana, em dias alternados, com posologia de $75 \mu \mathrm{g}$ de EGF em 
cada infiltração. Os efeitos adversos à utilização do protocolo foram pouco relatados, porém a segurança foi evidenciada.

Os resultados das aplicações foram: aumento no tecido de granulação, contração das bordas e reepitelização. Observou-se melhora no estresse oxidativo, aumento dos níveis das reservas antioxidantes, visto que a presença constante de estresse oxidativo no tecido aumenta a capacidade de danos celulares. Estes autores acreditam que essa capacidade do EGF em neutralizar o estresse oxidativo gera diminuição da apoptose prematura e promove a proliferação de fibroblastos locais, e é considerada uma terapia de reposição. Mesmo com a limitação do estudo, devido à baixa quantidade de participantes estudados, os autores acreditam que o aumento na granulação do tecido, contração das bordas da ferida e a reepitelização, se dão devido à diminuição no nível redox, dado por sua função redutora do estresse oxidativo.

Como resultado final o EGF mostrou-se eficaz assim como nos estudos anteriores, com a diferença que o autor mostra a melhora da cicatrização do PD. Gomez et al., 2014 em sua pesquisa também encontrou esta função de diminuição do estresse oxidativo do EGF, citando-o de forma mais superficial do que a pesquisa descrita.

Em uma revisão sistemática sobre FTC's utilizados por via tópica, Sridharane Sivaramakrishnan, 2017, trouxe os resultados de 26 ensaios clínicos, com 2088 participantes. Neste estudo, foi realizada a análise dos FTC's: rhEGF, PRP, rhPDGF, FGFb, rhVEGF e rhTGFß, concluindo que apenas os fatores rhEGF, rhPDGF e PRP, mostraram resultados concretos quanto a sua capacidade na estimulação da cicatrização do PD. Estes autores afïmam que o uso tópico dos FTC's auxilia na diminuição da celulite, da infecção, do edema e melhora a cicatrização.

Os demais fatores não possuíam a quantidade adequada para comparações ou os artigos estavam inconclusivos quanto ao resultado e/ou sua eficácia no tratamento. Como resultado observou-se que o rhEGF é mais eficaz que o fator PRP, e que este mesmo fator PRP mostrou-se mais efetivo na cicatrização quando comparado com o fator rhTGFß. Os fatores rhTGFß e PRP foram associados a redução de infecção na ferida. O rhTGFß também possui capacidade na redução da celulite. Os resultados acima citados corroboram com os estudos de Martí et al., 2015 e Gomez et al., 2014. Os autores destes artigos, informam em suas pesquisas, que o fator rhEGF auxilia na redução da dor e sensação de queimação na ferida do PD.

Os resultados dos estudos encontrados mostram a importância de novas pesquisas, com maior número de participantes, com dados dos efeitos adversos encontrados após a utilização destes agentes, para que se possa realizar um protocolo específico com maior segurança, significância e confiabilidade dos resultados obtidos.

\section{CONCLUSÃO}

Os fatores de crescimento são eficazes no tratamento do PD, sendo os mais estudados e citados: o fator de crescimento epidérmico (EGF) e o fator de crescimento 
recombinante humano (rhEGF). Mesmo com os resultados positivos quanto à aceleração e auxílio na cicatrização das feridas diabéticas, notou-se um déficit na apresentação das informações quanto ao protocolo, tempo de cicatrização, reincidência e a precisão da continuidade dos tratamentos cotidianos, o que leva à necessidade de novas pesquisas sobre os FTC's para que possa ser utilizado de forma isolada, mais segura e ainda mais eficiente.

\section{REFERÊNCIAS}

BRASIL. Ministério da Saúde. Secretaria de Atenção à Saúde. Departamento de Atenção Básica. Manual do pé diabético: estratégias para o cuidado da pessoa com doença crônica. Brasília: Ministério da Saúde, 2016.

ELISAVET, K.T.; NIKOLAOS, P.; ANASTASSIOS, C.M. and GEORGE, S.G. Epidermal Growth Factor in the Treatment of Diabetic Foot Ulcers: An Update. Perspectives in Vascular Surgery and Endovascular Therapy. Vol 24, Issue 1, pp. 37 - 44 First Published April 1 1, 2012. https://doi.org/10.1177/1531003512442093

ERTUGRUL, B.M.; LIPSKY, B.A.; GUVENC, U et al. An assessment of intralesional epidermal growth factor for treating diabetic foot woundsthe first experiences in Turkey. J Am Podiatr Med Assoc. 2017;107:17-29.

GOMEZ,V.R.; AGUILAR, R.F.; LOZANO, P.A.. et al. Efficacy of intralesional recombinant human epidermal growth factor in diabetic foot ulcers in Mexican patients: a randomized double-blinded controlled trial. Wound Repair Regen .2014;22:497-503.

MARTÍ C.A.J.; GLUUD, C.; NICOLA, S.; SIMANCAS, R.D; REVEIZ, L.; OLIVA, P.; CEDEÑO T.J. Growth factors for treating diabetic foot ulcers. Cochrane Database of Systematic Reviews 2015. Issue 10. Art. No.: CD008548. DOI: 00.1002/14651858. CDo08548.pub2.

MENEZES, M.M.; LOPES, C.T.; NOGUEIRA, L.S. Impact of educational interventions in reducing diabetic complications: a systematic review. Rev Bras Enferm [Internet]. 2016;69(4):726-37. DOI: http://dx.doi.org/10.1590/0034$7167.2016690422 \mathrm{i}$

OLIVEIRA C.M.; RODRIGUES, N.J.F.;SILVEIRA, M.F.; NEVES, D.M.R.; VILHENA, J.M.; OLIVEIRA, J.F. et al. The impact of risk factors of non-communicable chronic diseases on quality of life. Ciênc Saúde Colet [Internet]. 2013[cited 2014 Mar 01];18(3):873-82. 
OJALVO, A.G.; ACOSTA, J.B.; MARÍ, Y.M.; MAYOLA, M.F.; PÉREZ, C.V.; GUTIÉRREZ, W.S.; MARICHAL, II.; SEIJAS, E.Á.; KAUTZMAN, A.M.; PACHECO., A.E.; ARMSTRONG, D.G. Healing enhancement of diabetic wounds by locally infiltrated epidermal growth factor is associated with systemic oxidative stress reduction. Int Wound J. 2017 Feb; 14(1):2 14-225. doi: 10.1111/iwj.12592. Epub 2016 Mar 22.

PARISI, M.C.R. A síndrome do pé diabético, fisiopatologia e aspectos práticos. E-book 2.0 - Diabetes na prática Clínica (s.d.). Sociedade Brasileira de Diabetes. Disponível em: <http://www.diabetes.org.br/ebook/component/k2/item/42-asindrome-do-pe-diabeticofisiopatologia-e-aspectos-praticos $>>$. Acesso em: 31 maio. 2018.

PICARD, F.; HERSANT, B.; BOSC, R.; MENINGAUD, J.P. The growing evidence for the use of platelet-rich plasma on diabetic chronic wounds: A review and a proposal for a new standard care. Wound Repair Regen;23(5): 638-43, 2015 Sep.

SRIDHARAN, K.; SIVARAMAKRISHNAN, G. Growth factors for diabetic foot ulcers: Mixed treatment comparison analysis of randomized clinical trials. Br J Clin Pharmacol,2017 Nov 17.

YANG, S.; GENG, Z.; MA, K.; SUN, X.; FU, X. Efficacy of Topical Recombinant Human Epidermal Growth Factor for Treatment of Diabetic Foot Ulcer: A Systematic Review and Meta-Analysis. Int. J. Lower Extremity Wounds, 20 16, 15, 120-125 DOI: $10.1177 / 1534734616645444$ 